\title{
Reconfigurable RF-Waveform Generation Based on Incoherent-Filter Design
}

\author{
Víctor Torres-Company, Student Member, IEEE, Student Member, OSA, Jesús Lancis, Member, OSA, \\ Pedro Andrés, Fellow, OSA, and Lawrence R. Chen, Senior Member, IEEE, Member, OSA
}

\begin{abstract}
Radio-frequency (RF) waveform generators are key devices for a variety of applications, including radar, ultra-wideband communications, and electronic test measurements. Following advances in broadband coherent pulsed sources and pulse-shaping technologies, reconfigurable RF waveform generators operating at bandwidths $>1 \mathrm{GHz}$ have become a reality. In this work, we demonstrate reconfigurable $R F$ waveform generation using broadband spectrally incoherent optical sources. This is achieved in two steps. First, we implement an RF incoherent filter. The energy spectrum of the optical source is conveniently apodized using a commercially available computer-controlled D-WDM channel selector with $100-\mathrm{GHz}$ resolution. The channel controller provides high flexibility for shaping the optical source energy spectrum and, hence, high reconfigurability capabilities in terms of the RF filter. Second, we show that by applying a short baseband electrical waveform to the input of the RF filter, the output RF spectrum of the electrical signal is a mapped version of the designed RF filter transfer function. Specifically, we illustrate the capabilities of our technique by generating RF signals with $\sim 10 \mathrm{GHz}$ bandwidth and tunable repetition rate. Finally, we discuss how this method can be scaled up to the millimeter-wave range with current technology.
\end{abstract}

Index Terms-Microwave photonics, optical propagation in dispersive media, optical pulse shaping.

\section{INTRODUCTION}

$\mathbf{P}$ HOTONIC processing of microwave and radio-frequency (RF) signals is a research topic that has been explored for more than 30 years [1]-[3]. Indeed, microwave photonics offers many well-known advantages like reconfigurability, high bandwidth, immunity to electromagnetic interference, high-speed processing, and potential integrability with fiber

Manuscript received January 30, 2008; revised April 15, 2008. Current version published October 10, 2008. This work was supported by the Dirección General de Investigación Científica y Técnica, Spain, and FEDER under Project FIS2007-62217; the Convenio Universitat Jaume I-Fundació Caixa Castelló under Project P1 1B2007-09; and in part by the Natural Sciences and Engineering Research Council of Canada. V. Torres gratefully acknowledges financial assistance from a FPU grant of the Ministerio de Educacion y Ciencia, Spain.

V. Torres-Company and J. Lancis are with the Departament de Física, Universitat Jaume I, 12080 Castelló, Spain (e-mail: vtorres@ fca.uji.es; lancis@exp. uji.es).

P. Andrés is with the Departamento de Óptica, Universitat de València, 46100 Burjassot, Spain (e-mail: pedro.andres@uv.es).

L. R. Chen is with the Department of Electrical and Computer Engineering, McGill University, Montréal, QC H3A2A7 Canada (e-mail: lawrence.chen@mcgill.ca).

Color versions of one or more of the figures in this paper are available at http://ieeexplore.ieee.org.

Digital Object Identifier 10.1109/JLT.2008.925629 optics technology [4]. These features are very difficult, if not impossible, to achieve with electronic approaches. Typical RF signal processing applications cover analog-to-digital conversion, beamforming, filtering, and arbitrary waveform generation (AWG) [5].

In a photonic microwave filter, the input RF signal modulates an optical source, the modulated optical signal is then processed in the photonic domain, and finally, the RF signal is recovered back in the electrical domain by optoelectronic $(\mathrm{O} / \mathrm{E})$ conversion [4], [5]. Usually, incoherent discrete-time geometries are preferred because the filtering operation is more robust against environmental fluctuations [6]. It is then required that the coherence time of the optical source be shorter than the tap delay, ensuring that intensities are summed and weighted before O/E conversion. However, since intensity is always positive, the RF filter transfer function design cannot be completely arbitrary. From a digital-filter perspective, this is because the tap weight is positive. This drawback may be solved, e.g., by employing alternative modulation formats combined with differential photodetection [4], [7]-[11], nonlinear effects in either semiconductor optical amplifiers [12], or optical fibers [13], [14]. Additionally, in discrete-time processing, the resulting RF filter response is periodic. This feature can be partially overcome by apodizing the weight of the taps, which also permits to manipulate the shape of the first resonance [15]-[19].

On the other hand, with the recent advent of stable in-fiber frequency comb generators [20]-[22], availability of all-fiber supercontinuum sources [23], [24], and the development of ultrahigh resolution pulse shapers [25]-[27], reconfigurable RF waveform generators have been proposed and experimentally demonstrated [28]-[33]. In general terms, the upper limit in terms of the analog RF spectral content that can be processed using photonic approaches is set by the $\mathrm{O} / \mathrm{E}$ conversion bandwidth. Commercially available photodiodes currently place this limit beyond the millimeter-wave region. As a consequence, photonically assisted RF-AWG constitutes a reliable alternative to its electronic counterpart, whose state-of-the-art RF bandwidth is restricted to $<5 \mathrm{GHz}$ due to the limited speed of analog-to-digital conversion. Among the many different architectures reported in the literature, those based on Fourier-transform pulse shapers are the most popular ones [29], [32], [33]. In these configurations, the spectrum of a coherent broadband source, e.g., a mode-locked laser or a supercontinuum light source, is tailored in a zero-dispersion pulse shaper and stretched temporally in a dispersive medium. This way, a frequency-to-time mapping [34] takes place, i.e., the photodetected output intensity is a scaled replica of the 
synthesized optical spectrum. In a first-order approximation, the scale factor is given by the group-delay-dispersion (GDD) coefficient of the stretching element [35]. The advantages of this technique are the inherent reconfigurability due to the spatial light modulator employed to shape the optical spectrum, the possibility to create a feedback loop which corrects distortions in the achieved waveform [36], and intrinsic fiber transport if a single-mode fiber (SMF) is employed as the dispersive medium. Despite these enormous benefits, the low repetition rate of passively mode-locked lasers, the high equipment cost, and the need for nonlinear effects to enhance the optical spectrum motivate the need for alternative solutions to perform photonic RF-AWG.

Recently, we theoretically proposed a reconfigurable RF waveform generator based on a Fourier transform geometry operating with an incoherent light source [37]. Here, we provide an extended analysis of our preliminary experimental results [38] and, in particular, analyze the performance of the system in terms of an incoherent RF filtering process. This interpretation suggests that fully arbitrary RF signal generation can be achieved simply with incoherent sources by using different modulation architectures. Here, we achieve RF analog signals with $\sim 10-\mathrm{GHz}$ bandwidth at tunable repetition rates. Moreover, we analyze the theoretical limits of our technique and show that it can be scaled to the millimeter-wave range using conventional technology.

The remainder of this paper is organized as follows. In Section II, we describe the specific experimental arrangement. This is followed by a first-order analysis in Section III. We show that the experimental setup can be interpreted as an incoherent filter, whose energy spectrum (ES) is tailored in a continuous manner, i.e., the tap weight is windowed. Although this does not avoid the above-mentioned problem of using only positive weight taps, the system's reconfigurability and spectral resolution allow us to achieve a variety of interesting waveforms for a broad range of applications. In Section IV, we show some examples of the measured RF filter transfer functions, and in Section $\mathrm{V}$, we show the capabilities of the system to achieve reconfigurable RF signal generation. Finally, in Section VI, we highlight the main advantages and drawbacks of our technique and discuss the fundamental issues that need to be addressed for scaling up to the millimeter-wave range.

\section{Set-Up Overview}

Our experimental setup for RF-AWG is represented schematically in Fig. 1. We use the amplified spontaneous emission light from an Erbium-doped fiber amplifier (EDFA) as a broadband incoherent source. The ES is then tailored (shaped) using a commercially available D-WDM 48-channel controller with $100-\mathrm{GHz}$ resolution spanning the whole $\mathrm{C}$ band and $<6 \mathrm{~dB}$ insertion losses (Peleton, QTM). The channel controller consists of a zero-dispersion pulse shaper [25] with two diffractive gratings as angular dispersive elements and a spatial light modulator for amplitude control. The amplitude of each channel can be blocked fully or reduced in increments of $0.1 \mathrm{~dB}$ up to a maximum of $20 \mathrm{~dB}$. Due to the source incoherence, the output waveform is independent of the reminiscent spectral phase of the individual channels [39]. After ES synthesis, the light is polar-

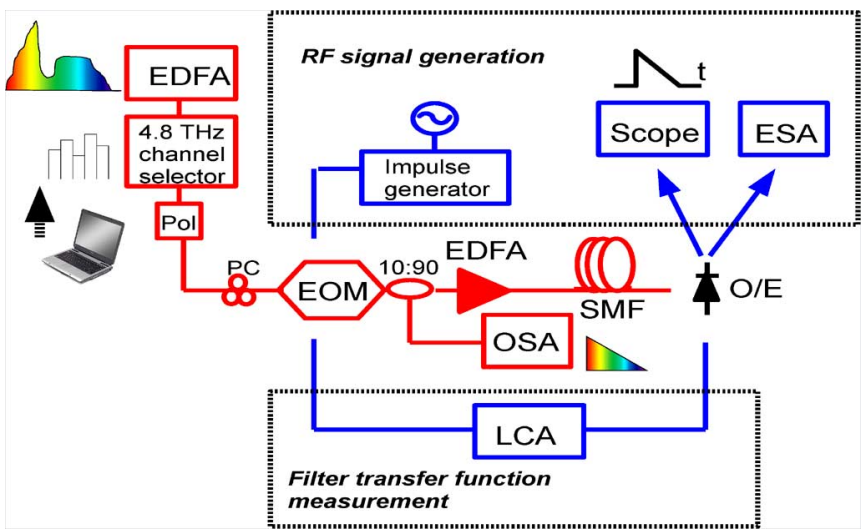

Fig. 1. Experimental setup. Lower dash-dotted box is used for the measurement of the system's transfer function, whereas the upper box is used for the RF signal generation. Acronyms are explained in the text.

ized before entering an external modulator in order to increase the extinction ratio of the modulated signal at the output. The modulator is a simple $10-\mathrm{Gb} / \mathrm{s} \mathrm{LiNbO} 3$ electro-optic modulator (EOM) biased at quadrature. The modulated optical signal is amplified and launched into a coil of SMF. Finally, the optical signal is converted back to the electrical domain using a photodiode. The electrical RF filter transfer function can be defined between the input and output RF signals [6], [15]. The measurement of this transfer function is performed using a lightwave component analyzer (LCA) (see the lower box in Fig. 1).

Once the transfer function is synthesized adequately, we remove the LCA and drive the EOM with an electrical pulse with a full width half maximum (FWHM) as short as 60 ps (upper box in Fig. 1) from an impulse signal generator (Picosecond Pulse Labs, 3600). The impulse signal generator is driven by a clock signal from an external CW generator with a frequency range between $10 \mathrm{MHz}$ to $20 \mathrm{GHz}$. This permits the tuning of the repetition rate so that CW RF waveforms can be easily achieved. At the output of the fiber, the signal is measured either in the time domain using a digital scope with an optical sampling module having a bandwidth of $20 \mathrm{GHz}$ or in the spectral domain using a photodiode with a 45-GHz bandwidth connected to an electrical spectrum analyzer (ESA).

\section{FIRST-ORDER THEORY}

Based on the system shown in Fig. 1, if the input RF signal entering the EOM does not induce higher order harmonic distortion and the photodiode behaves linearly, then there will be a linear relation between the input RF signal and the output RF signal from the photodiode [6], [15]. The corresponding filter transfer function $H(f)$ can be shown to be (see [19] for a detailed analysis based on coherence theory concepts)

$$
H(f)=\cos \left(2 \pi^{2} \Phi_{2} f^{2}\right) \Gamma_{s}\left(-2 \pi \Phi_{2} f\right) .
$$

$\Phi_{2}$ is the GDD parameter, which for the case of SMF is $\Phi_{2}=$ $\beta_{2} z$, where $\beta_{2}$ is the group velocity dispersion coefficient, and $z$ is the fiber length. The first term in (1) corresponds to the double-sideband (DSB) modulation inherent to intensity modulators. Alternative modulation formats, such as single-sideband 
or phase modulation, lead to different expressions. The second term is the mutual coherence function (MCF) of the apodized incoherent optical source, which is related to the ES through an inverse Fourier transform (known as Wiener-Khintchine theorem [40]). Once the ES is synthesized, $H(f)$ remains fixed for a given $\Phi_{2}$. Consequently, the electrical spectrum (in amplitude and phase) of any RF signal input will be filtered by $H(f)$. We note that similar arrangements with spatial light modulators or microelectromechanical mirrors have been reported recently [15], [18], [41]. Our objective is to employ such an RF filter to perform reconfigurable RF waveform generation.

With this aim, let us assume a baseband RF signal with input spectrum $\widetilde{I}_{i n}(f)$ driving the EOM. If the following conditions are satisfied:

$$
\sigma_{0} \gg t_{c}
$$

and

$$
\Phi_{2} \gg \sigma_{0} t_{c} /(2 \pi)
$$

where $\sigma_{0}$ is the electrical temporal pulse width of the input RF signal and $t_{c}$ is the coherence time of the synthesized ES, the output electrical spectrum $\widetilde{I}_{\text {out }}(f)$ will be roughly

$$
\tilde{I}_{\text {out }}(f) \approx \Gamma_{s}\left(-2 \pi \Phi_{2} f\right)
$$

By inverse Fourier transformation, we get

$$
I_{\text {out }}(t) \approx S\left(\frac{t}{\Phi_{2}}\right) .
$$

Here, $S(\omega)$ denotes the ES of the synthesized incoherent source. Equations (4) and (5) are complementary: Equation (4) can be interpreted as a mapping from the MCF to the electrical RF spectrum, whereas (5) is an incoherent frequency-to-time mapping, i.e., the output RF signal in time is a scaled replica of the synthesized ES [42]. It is important to understand that by varying the GDD coefficient, we can tune the RF bandwidth of the electrical signal. In this sense, we have the same features as in RF waveform generators based on the Fourier transform pulse shaper, i.e., once the wavelength-to-time mapping is achieved, increasing the GDD does not alter the RF waveform shape: only the scale.

From (1), we note that, in general, the filter transfer function is given by the multiplication of two terms. The first function presents a relatively flat behavior in the region $0<f<f_{c}$, where $f_{c}$ is the first cutoff frequency, i.e., $f_{c}=\left(2 \sqrt{\pi \Phi_{2}}\right)^{-1}$. The second function has an RF width $\Delta f$, that is given by a scaled version of the coherence time of the synthesized MCF, $\Delta f=t_{c} /\left(2 \pi \Phi_{2}\right)$. Therefore, when conditions in (2) and (3) are satisfied, the term $\Gamma_{s}$ dominates the structure of $H(f)$. Thus, the proper synthesis of $\Gamma_{s}$, through the tailoring of the ES with the D-WDM channel controller, gives the reconfigurability capabilities. However, since the ES is always real and positive, $\Gamma_{s}$ is Hermitian and maximum at origin. This implies that fully arbitrary filter transfer functions, and hence arbitrary output electrical signals, cannot be achieved with this configuration. Never-

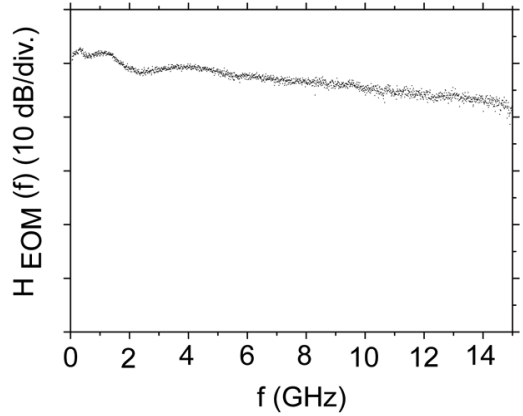

Fig. 2. Transfer function term provided by the EOM.

theless, with a proper design of the ES, a broad range of practical waveforms can be generated.

\section{FILTER TRANSFER FUnCTION MEASUREMENT}

As mentioned in the previous section, the system shown in Fig. 1 can be used to implement a linear filter for an RF signal at the input of the EOM. In this section, we demonstrate this experimentally and show the measured filter transfer function for some particular examples. According to (1), the filter transfer function is determined by two terms. By properly selecting the GDD amount, the impact of the first term corresponding to the DSB modulation can be neglected. In this case, the filter response will be given by the MCF of the source. To show this, we first use a narrow linewidth $\mathrm{CW}$ laser as the optical source and measure the filter transfer function with the LCA for a fiber length of $4.6 \mathrm{~km}$; see Fig. 2. For this fiber length, the first zero of the first term in (1) occurs at $28 \mathrm{GHz}$, which is beyond our range of interest. In particular, we can appreciate that the contribution of the DSB modulation is relatively flat, and within a good approximation, we can state that the term corresponding to $\Gamma_{s}$ will determine $H(f)$.

We now replace the $\mathrm{CW}$ laser with the incoherent source filtered using the channel controller. It is important to note that the taps provided by the channel controller allow for a high resolution windowing because the slicing is performed in a continuous manner (though with finite resolution). To show this, we first synthesize a "discrete" ES illustrated in Fig. 3(a), which comprises five taps, where each are $0.8 \mathrm{~nm}$ wide and separated by 1.6 $\mathrm{nm}$, and with different power. The corresponding filter transfer function for $4.6 \mathrm{~km}$ of SMF is then measured by sweeping the LCA from $50 \mathrm{MHz}$ to $15 \mathrm{GHz}$. The filter amplitude and group delay responses are shown in Figs. 3(b) and (c), respectively. For this designed ES, we obtain a resonant bandpass centered at $8.4 \mathrm{GHz}$ with a $3-\mathrm{dB}$ bandwidth of $1.5 \mathrm{GHz}$. We also observe the typical sidelobes arising from the periodic nature of the ES. As a second example, we tailor the ES in a more "continuous" manner, as shown in Fig. 3(d). In this case, we obtain a flat-top RF filter that spans over the frequency range from 3.2 to $8.8 \mathrm{GHz}$, which is compatible with the ultra-wideband (UWB) specifications from 3.1 to $10.6 \mathrm{GHz}$; see Figs. 3(e) and (f).

The above examples illustrate the capabilities and main drawbacks of our technique. We can synthesize an RF filter spanning a range from 0 to $10 \mathrm{GHz}$. The upper limit is set partially by the response of the EOM, and higher values can be obtained using 

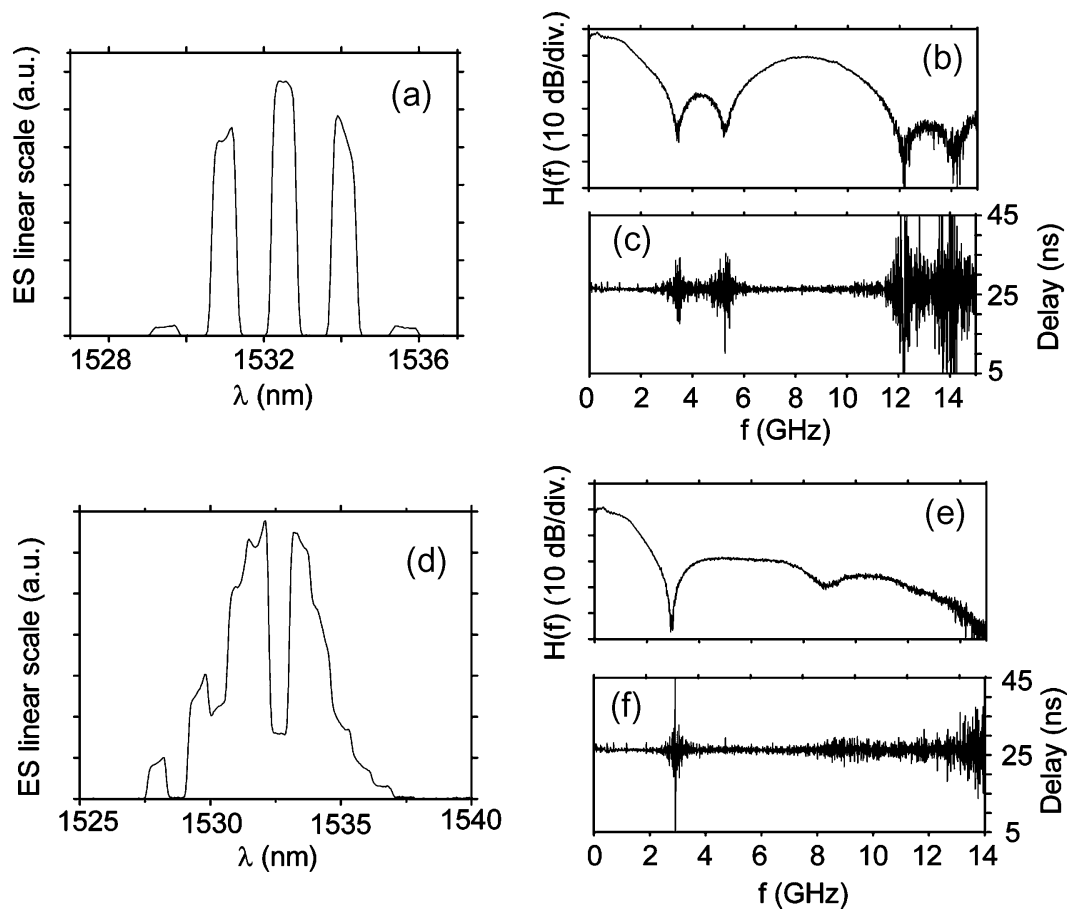

Fig. 3. (a) Optical ES of a tapped incoherent source in a discrete-time geometry. (b) Resulting filter amplitude shape when an SMF of $4.6 \mathrm{~km}$ length is employed as stretching element. (c) Delay response of the filter. (d) Second apodized incoherent ES. (e) Resulting filter amplitude shape for the same fiber length. (f) Delay response of the filter.

higher bandwidth modulators. The baseband resonance that appears is inherent to DSB modulation and square-law photodetection. In other words, we can implement an incoherent filter with positive coefficients only. The role of the channel controller is to modify the ES so that a second RF passband can be tuned and tailored. The spectral window $(4.8 \mathrm{THz}$ in our case, corresponding to the bandwidth of the channel controller) determines the minimum resolvable $\mathrm{RF}$ frequency, i.e., $\left(4.8 \mathrm{THz} * \Phi_{2}\right)^{-1}$. Conversely, the minimum spectral feature in the setup $(100 \mathrm{GHz}$ corresponding to the resolution of the controller) imposes an RF spectral window of $\left(0.1 \mathrm{THz} * \Phi_{2}\right)^{-1}$.

\section{RECONFIGURABLE RF Signal GENERATION}

Once the filter transfer function is properly designed (by tailoring the ES), an RF signal generator can be implemented simply by inserting into the system an input baseband broadband electrical waveform. In our case, we use a Gaussian electrical pulse with a FWHM duration of 60 ps as the broadband input. We must note that the available optical spectral width of $4.8 \mathrm{THz}$ leads to a coherence time of $33 \mathrm{fs}$ so that the condition specified in (2) is satisfied. Even for the case in which one channel is passed and the rest are blocked, the resulting coherence time (1.6 ps) also satisfied the condition described in (2).

Fig. 4 shows a numerical plot of the RF spectrum of the input electrical pulse. Since the amplitude is relatively flat for the frequency region $<10 \mathrm{GHz}$, the output $\mathrm{RF}$ spectrum will be given essentially by the filter response $H(f)$. Assuming further that the condition specified in (3) is also satisfied, the filter response is determined by the synthesized MCF of the optical source.

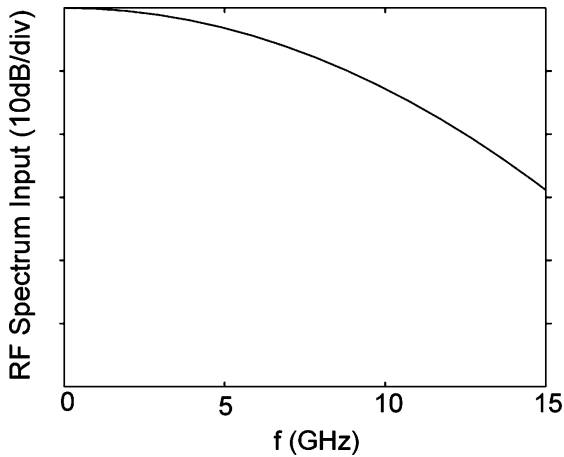

Fig. 4. RF spectrum of the input electrical pulse.

The first example concerns the generation of a sawtooth pulse. Such a waveform has been generated previously using a Fourier transform pulse shaper [43] or a fiber grating passive device [44]; in both of these cases, a broadband coherent pulsed source was required. The synthesized ES is shown in Fig. 5(a). After temporal modulation and stretching in $7.6 \mathrm{~km}$ of SMF, the measured temporal waveform is shown in Fig. 5(b) and (c) in sample mode with $100 \mathrm{~ms}$ persistence and with four times averaging, respectively. The incoherent frequency-to-time mapping predicted by (5) is clearly visible. The RF spectrum obtained when using a 4.6-km length of SMF and $100-\mathrm{MHz}$ repetition rate is shown in Fig. 5(d). The second example corresponds to the generation of a short pulse burst. The synthesized ES is shown in Fig. 5(e); the temporal waveform after stretching in $7.6 \mathrm{~km}$ of SMF is shown in Fig. 5(f) in sample mode and 5(g) with four times averaging. Fig. 5(h) shows the measured RF spectrum when $4.6 \mathrm{~km}$ of SMF is used, as well as a repetition 

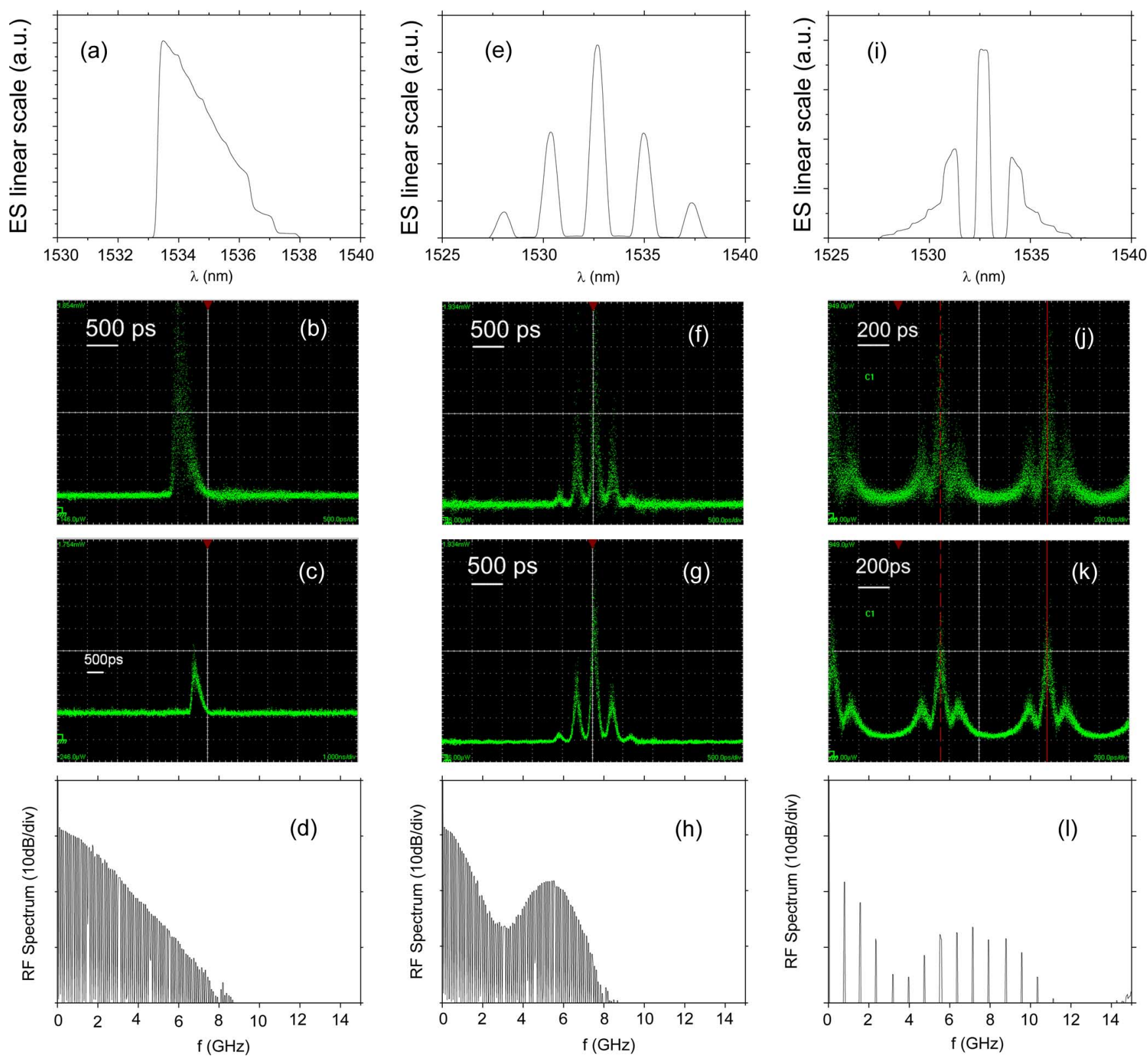

Fig. 5. Three different single pulse RF signals. Each example corresponds to each column. First row [(a), (e), and (i)] corresponds to the ES. Second [(b), (f) and (j)] and third rows [(c), (g), and (k)] correspond to time intensity in linear arbitrary scale in sample and four times averaged, respectively. Last row [(d), (h), and (1)] correspond to the RF spectra. The specific settings are explained in the text.

rate of $300 \mathrm{MHz}$. The final example concerns the generation of a doublet-like pulse. Fig. 5(i) shows the ES, and Fig. 5(j) and $(\mathrm{k})$ shows the temporal waveform in sample mode and with four times averaging, respectively, when using $4.6 \mathrm{~km}$ of SMF. The corresponding RF spectrum is shown in Fig. 5(1) for a repetition rate of $800 \mathrm{MHz}$.

One of the key advantages of the proposed technique is the possibility to control the repetition rate of the generated waveform in an easy way. This is achieved by changing the clock frequency that drives the electrical impulse generator. Thus, we can obtain continuous RF waveforms. In particular, we illustrate the generation of a CW sawtooth waveform and a chirped sinusoidal waveform, both with four times averaging, in Fig. 6(a) and $6(\mathrm{~b})$, respectively.

\section{SUMMARY AND DISCUSSIONS}

In summary, we have demonstrated a reconfigurable RF waveform generator that operates with a broadband spectrally incoherent source. The implementation of this device is based on the proper design of an incoherent microwave filter with positive coefficients [15]. The tap weight is conveniently adjusted using a commercial D-WDM 48-channel controller. By applying a short RF pulse (broadband input) to the synthesized RF filter, the output electrical signal (in frequency) is given by a scaled replica of the MCF of the ES of the incoherent source. Alternatively, this constitutes an incoherent frequency-to-time mapping [37].

Since the incoherent filter has been implemented with positive coefficients only, the range of achievable RF signals is 

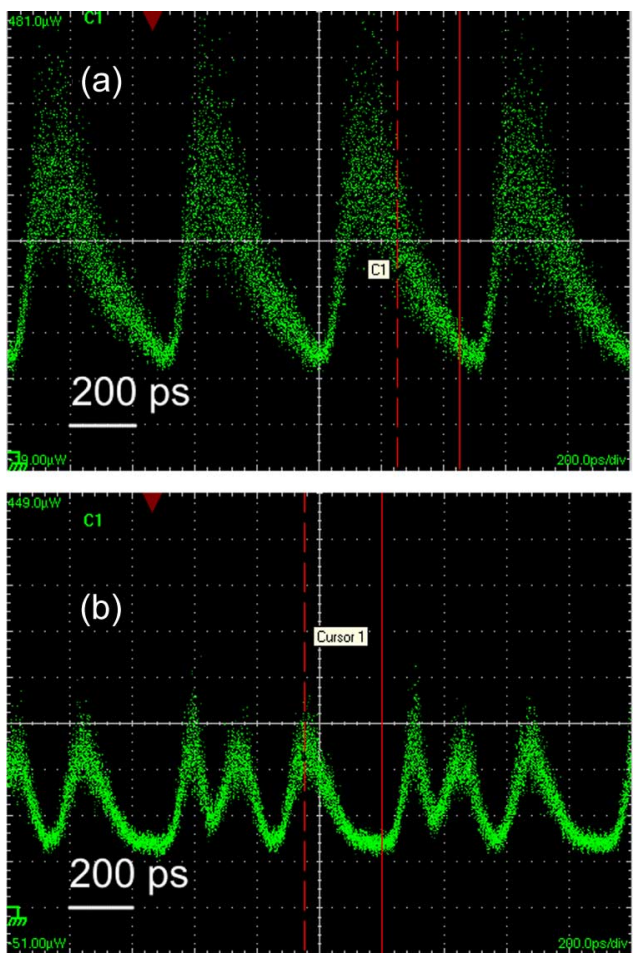

Fig. 6. CW RF waveforms. (a) Sawtooth pulse. (b) Chirped sinusoidal profile.

limited, which makes our RF synthesizer, as well as those based on Fourier transform pulse shaping and frequency-to-time mapping, not strictly arbitrary. However, thanks to the multiple-tap geometry, many practical waveforms can be achieved. Truly RF-AWG could be implemented by designing a proper microwave-photonic filter with user-defined positive, negative, or complex coefficients in a multitap geometry [45]. One of the key advantages of our technique is that the repetition rate of the generated RF pulse can be controlled easily, which allows us to achieve even continuous waveforms.

Once the incoherent frequency-to-time mapping is achieved, the output waveform can be scaled by increasing the amount of GDD. This leads to decreasing the achievable RF bandwidth. In our experiments, the upper limit is approximately $10 \mathrm{GHz}$. This limit is not a fundamental constraint and is set by both the EOM and the pulse duration provided by the electrical impulse generator. By using shorter electrical signals, a high bandwidth electroabsorption modulator, and the same D-WDM channel selector, our technique can be scaled up to operate in the millimeter-wave range. In particular, Fig. 7 gives a rough estimation of the maximum achievable electrical bandwidth in terms of the input electrical pulse width. By using an external modulator providing $<20 \mathrm{ps}$ temporal widths, it is possible to achieve operation in the millimeter-wave region.

Finally, we must emphasize that the original configuration of the technique proposed in [37] is not necessarily based on a microwave filter design. To achieve the incoherent frequency-totime mapping, it is just required to modulate the synthesized ES.

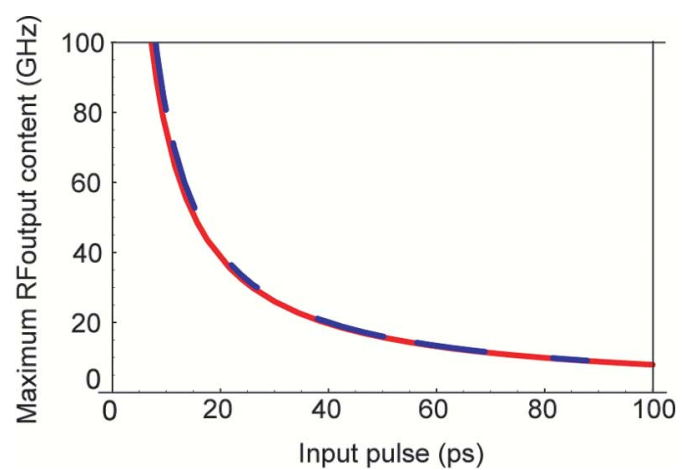

Fig. 7. Maximum achievable RF electrical bandwidth as a function of the input light pulse duration. Solid curve is for a single channel on and dashed curve for all channels on.

\section{ACKNOWLEDGMENT}

The authors acknowledge Peleton Corporation for the loan of the channel controller. V. Torres and P. Andrés thank Dr. J. Cordero for his kind assistance with the RF spectra measurements.

\section{REFERENCES}

[1] K. Wilner and A. P. V. den Heuvel, "Fiber-optic delay lines for microwave signal processing," Proc. IEEE, vol. 64, no. 5, pp. 805-807, May 1976.

[2] K. Jackson, S. Newton, B. Moslehi, M. Tur, C. C. Cutler, J. W. Goodman, and H. J. Shaw, "Optical fiber delay-line signal processing," IEEE Trans. Microw. Theory Techn., vol. MTT-33, no. 3, pp. 193-204, Mar. 1985.

[3] A. Seeds, "Microwave photonics," IEEE Trans. Microw. Theory Techn., vol. 50, no. 3, pp. 877-887, Mar. 2002.

[4] J. Capmany and D. Novak, "Microwave photonics combines two worlds," Nature Photon., vol. 1, no. 6, pp. 319-330, Jun. 2007.

[5] R. A. Minasian, "Photonic signal processing of microwave signals," IEEE Trans. Microw. Theory Tech., vol. 54, no. 2, pp. 832-846, Feb. 2006.

[6] J. Capmany, B. Ortega, D. Pastor, and S. Sales, "Discrete-time optical processing of microwave signals," IEEE/OSA J. Lightwave Technol., vol. 23, no. 2, pp. 702-723, Feb. 2005.

[7] S. Sales, J. Capmany, J. Martí, and D. Pastor, "Experimental demonstration of fiberoptic delay-line filters with negative coefficients," Electron. Lett., vol. 31, no. 13, pp. 1095-1096, Jun. 1995.

[8] J. Capmany, D. Pastor, A. Martínez, B. Ortega, and S. Sales, "Microwave photonic filters with negative coefficients based on phase inversion in an electro-optic modulator," Opt. Lett., vol. 28, no. 16, pp. 1415-1417, Aug. 2003.

[9] F. Zeng and J. P. Yao, "All-optical microwave filter based on an electrooptic phase modulator," Opt. Express, vol. 12, no. 16, pp. 3814-3819, Aug. 2004.

[10] N. S. You and R. A. Minasian, "All-optical photonic signal processors with negative coefficients," IEEE/OSA J. Lightwave Technol., vol. 22, no. 12, pp. 2739-2742, Dec. 2004.

[11] J. Mora, J. Capmany, A. Loayssa, and D. Pastor, "Novel technique for implementing incoherent microwave photonic filters with negative coefficients using phase modulation and single sideband selection," IEEE Photon. Technol. Lett., vol. 18, no. 17, pp. 1943-1945, Sept. 2006

[12] F. Coppinger, S. Yegnanarayan, P. D. Trinh, and B. Jalali, "All-optical incoherent negative taps for photonic signal processing," Electron. Lett., vol. 33, no. 11, pp. 973-975, May 1997.

[13] A. Loayssa, J. Capmany, M. Sagues, and J. Mora, "Demonstration of incoherent microwave photonic filters with all-optical complex coefficients," IEEE Photon. Technol. Lett., vol. 18, no. 13, pp. 1744-1746, Jul. 2006.

[14] B. Vidal, M. A. Piqueras, and J. Martí, "Tunable and reconfigurable microwave filter based on stimulated Brillouin scattering," Opt. Lett., vol. 32, no. 1, pp. 23-25, Jan. 2007. 
[15] J. Capmany, J. Mora, D. Pastor, and B. Ortega, "High-quality onlinereconfigurable microwave photonic transversal filter with positive and negative coefficients," IEEE Photon. Technol. Lett., vol. 17, no. 12, pp. 2730-2732, Dec. 2005.

[16] B. Vidal, M. A. Piqueras, and J. Martí, "Photonic microwave filter based on spectrum slicing with reconfiguration capability," Electron. Lett., vol. 41, no. 23, pp. 1286-1287, Nov. 2005.

[17] J. Mora, B. Ortega, A. Díez, J. L. Cruz, M. V. Andrés, J. Capmany, and D. Pastor, "Photonic microwave tunable single-bandpass filter based on a Mach-Zender interferometer," IEEE/OSA J. Lightwave Technol., vol. 24, no. 7, pp. 2500-2509, Jul. 2006.

[18] J. H. Lee, Y. M. Chang, Y.-G. Han, S. B. Lee, and H. Y. Chung, "Fully reconfigurable photonic microwave transversal filter based on digital micromirror device and continuous-wave, incoherent supercontinuum source," Appl. Opt., vol. 46, no. 22, pp. 5158-5166, Aug. 2007.

[19] V. Torres-Company, J. Lancis, and P. Andrés, "Flat-top ultra-wideband photonic filters based on mutual coherence function synthesis," Opt. Commun, vol. 281, pp. 1438-1444, Mar. 2008.

[20] K. Imai, M. Kourogi, and M. Ohtsu, "30-THz span optical frequency comb generation by self-phase modulation in an optical fiber," IEEE J. Quantum Electron., vol. 34, no. 1, pp. 54-68, Jan. 1998.

[21] H. Murata, A. Morimoto, T. Kobayashi, and S. Yamamoto, "Optical pulse generation by electrooptic-modulation method and its application to integrated ultrashort pulse generators," IEEE J. Sel. Top. Quantum Electron., vol. 6, no. 6, pp. 1325-1331, Nov. 2000.

[22] T. Sakamoto, T. Kawanishi, and M. Izutsu, "Asymptotic formalism for ultraflat optical frequency comb generation using a Mach-Zender modulator," Opt. Lett., vol. 32, no. 11, pp. 1515-1517, Jun. 2007.

[23] T. Morioka, K. Mori, S. Kawanishi, and M. Saruwatari, "MultiWDM-channel, Gbit/s pulse generation from a single laser source utilizing LD-pumped supercontinuum in optical fibers," IEEE Photon. Technol. Lett., vol. 6, no. 3, pp. 365-368, Mar. 1994.

[24] O. Boyraz, J. Kim, M. N. Islam, F. Coppinger, and B. Jalali, "10 $\mathrm{Gb} / \mathrm{s}$ multiple wavelength, coherent short pulse source based on spectral carving of supercontinuum generated in fibres," IEEE/OSA J. Lightwave Technol., vol. 18, no. 22, pp. 2167-2175, Dec. 2000.

[25] A. M. Weiner, "Femtosecond pulse shaping using spatial light modulators," Rev. Sci. Inst., vol. 71, no. 5, pp. 1929-1960, May 2000.

[26] Z. Jiang, D. S. Deo, D. E. Leaird, and A. M. Weiner, "Spectral line-by-line pulse shaping," Opt. Lett., vol. 30, no. 12, pp. 1557-1559, Jun. 2005

[27] Z. Jiang, C. B. Huang, D. E. Leaird, and A. M. Weiner, "Optical arbitrary waveform processing of more than 100 spectral comb lines," Nature Photon., vol. 1, no. 8, pp. 463-467, Aug. 2007.

[28] J. D. McKinney, D. E. Leaird, and A. M. Weiner, "Millimeter-wave arbitrary waveform generation with a direct space-to-time pulse shaper," Opt. Lett., vol. 27, no. 15, pp. 1345-1347, Aug. 2002.

[29] J. Chou, Y. Han, and B. Jalali, "Adaptive RF-photonic arbitrary waveform generator," IEEE Photon. Technol. Lett., vol. 15, no. 4, pp. 581-583, Apr. 2003.

[30] P. J. Delfyett, S. Gee, M. T. Choi, H. Izadpanah, W. Lee, S. Ozharar, F. Quinlan, and T. Yilmaz, "Optical frequency combs from semiconductor lasers and applications in ultrawideband signal processing and communications," IEEE/OSA J. Lightwave Technol., vol. 24, no. 7, pp. 2701-2719, Jul. 2006.

[31] S. J. Xiao and A. M. Weiner, "Coherent Fourier transform electrical pulse shaping," Opt. Express, vol. 14, no. 7, pp. 3073-3082, Apr. 2006.

[32] B. Bortnik, I. Y. Poberezhskiy, J. Chou, B. Jalali, and H. R. Fetterman, "Predistortion technique for RF-photonic generation of high-power ultrawideband arbitrary waveforms," IEEE/OSA J. Lightwave Technol., vol. 24, no. 7, pp. 2752-2759, Jul. 2006.

[33] J. D. McKinney, I. S. Lin, and A. M. Weiner, "Shaping the power spectrum of ultra-wideband radio-frequency signals," IEEE Trans. Microw. Theory Techn., vol. 54, no. 12, pp. 4247-4255, Dec. 2006.

[34] Y. C. Tong, L. Y. Chan, and H. K. Tsang, "Fibre dispersion or pulse spectrum measurement using a sampling oscilloscope," Electron. Lett., vol. 33, no. 11, pp. 983-985, May 1997.

[35] M. A. Muriel, J. Azaña, and A. Carballar, "Real-time Fourier transformer based on fiber gratings," Opt. Lett., vol. 24, no. 1, pp. 1-3, Jan. 1999.

[36] I. S. Lin, J. D. McKinney, and A. M. Weiner, "Photonic synthesis of broadband microwave arbitrary waveforms applicable to ultra-wideband communication," IEEE Microw. Wire. Comp. Lett., vol. 15, no. 4, pp. 226-228, Apr. 2005.
[37] V. Torres-Company, J. Lancis, and P. Andrés, "Arbitrary waveform generator based on all-incoherent pulse shaping," IEEE Photon. Technol. Lett., vol. 18, no. 21, pp. 2626-2628, Nov. 2006.

[38] V. Torres-Company, J. Lancis, P. Andrés, and L. R. Chen, "Reconfigurable RF waveform generation using optical incoherent sources," in CLEO 2008, submitted for publication.

[39] L. J. Wang, B. E. Magill, and L. Mandel, "Propagation of thermal light through a dispersive medium," J. Opt. Soc. Amer. B, vol. 6, no. 5, pp. 964-966, May 1989.

[40] L. Mandel and E. Wolf, Optical Coherence and Quantum Optics. Cambridge, U.K.: Cambridge Univ. Press, 1995.

[41] N. A. Riza and M. A. Arain, "Programmable broadband radio-frequency transversal filter with compact fiber-optics and digital microeletromechanical system-based optical spectral control," Appl. Opt., vol. 43, no. 15, pp. 3159-3165, May 2004.

[42] C. Dorrer, "Temporal van Cittert-Zernike theorem and its application to the measurement of chromatic dispersion," J. Opt. Soc. Amer. B, vol. 21, no. 8, pp. 1417-1423, Aug. 2004.

[43] I. Y. Poberezhskiy, B. Bortnik, J. Chou, B. Jalali, and H. R. Fetterman, "Serrodyne frequency translation of continuous optical signals using ultra-wideband electrical sawtooth waveforms," IEEE J. Quantum Electron., vol. 41, no. 12, pp. 1533-1539, Dec. 2005.

[44] M. Shen and R. A. Minasian, "Toward a high-speed arbitrary waveform generation by a novel photonic processing structure," IEEE Photon. Technol. Lett., vol. 16, no. 4, pp. 1155-1157, Apr. 2004.

[45] M. Sagues, R. G. Olcina, A. Loayssa, S. Sales, and J. Capmany, "Multi-tap complex coefficient incoherent microwave photonic filters based on optical single-sideband modulation and narrow band optical filtering," Opt. Express, vol. 16, no. 1, pp. 295-303, Jan. 2008.

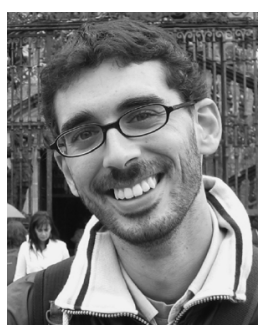

Víctor Torres-Company (M'06) was born in Valencia, Spain, in 1981. He received the B.Sc. degree in physics and the M.Sc. degree in photonics from University of Valencia in 2004 and 2006, respectively. He is currently a research member with the Physics Department at University Jaume I, Castelló, Spain, where he is pursuing the Ph.D. degree in physics.

$\mathrm{He}$ has research with the Royal Institute of Technology (KTH), Stockholm, Sweden, and the Technical University of Denmark (DTU), Copenhagen, Denmark. His main research interests are scalar coherence theory, pulse processing based on the space-time analogy, microwave photonics, and temporal entanglement phenomena.

Mr. Torres-Company is a student member of the OSA.

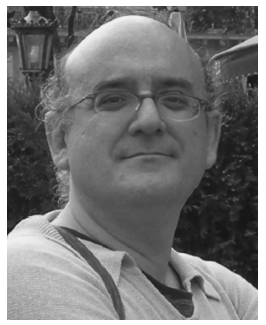

Jesús Lancis was born on December 30, 1965, in San Miguel de Salinas, Alicante, Spain. He received the B.Sc. degree in physics in 1988 and the M.Sc. and Ph.D. degrees in optics from the Universitat de València, Valencia, Spain, in 1989 and 1992, respectively.

From 1991 to the present, he has been with the Grup de Recerca d'Òptica de Castelló, Physics Department, Universitat Jaume I, Castelló, Spain. He has been a visiting scientist at both the University of North Carolina at Charlotte and the University of Minnesota, Minneapolis, where he worked in the field of diffractive optics. His current research interests include short pulse generation, pulse shaping, microwave photonics, femtosecond optics, liquid crystal displays, ophtalmic optics, and entangled photons. He is the author and/or co-author of more than 60 papers in leading optics journals.

Dr. Lancis is a member of Spanish Optical Soceity and the Optical Society of America. 


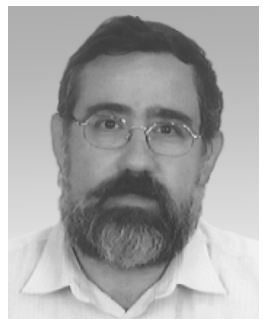

Pedro Andrés was born in Valencia, Spain, in 1954. $\mathrm{He}$ received the Ph.D. degree in physics/optics from the University of Valencia in 1983.

He has held a professorship of optics in the University of Valencia since 1994, where he was an associate professor from 1986 to 1993 . He also held the position of Head of the Department of Optics at the same university from 1998 to 2006 . He has carried out research as visiting scientist with the Laboratory of Optics P. M. Duffieux at the University of FrancheComté, Franche-Comté, France; with the National Institute for Astrophysics, Optics, and Electronics (INAOE), Mexico; with the National Physical Laboratory (NPL), U.K.; with the Department of Electrical and Computer Engineering, Pennsylvania State University, State College; with the University Jaume I, Castellón, Spain, and with the Department of Electrical and Computer Engineering, McGill University, Montreal, QC, Canada. His current research interests include wavelength compensation of light diffraction, propagation of periodic fields, image formation and scanning microscopy, modeling and design of photonic crystal fibers, temporal optics, and ultrafast optics. He has published more than 100 original papers subject to peer review and he has presented 27 invited papers and more than 110 contributions in international conferences and meetings.

Dr. Andrés is Fellow of the Optical Society of America (OSA).

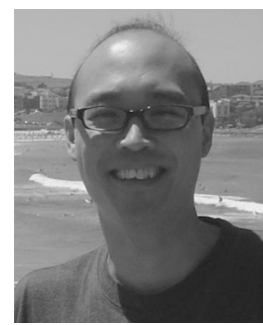

Lawrence R. Chen (M'00-SM'06) was born on February 17, 1973, in Red Deer, AB, Canada. He received the B.Eng. degree in electrical engineering and mathematics from McGill University, Montreal, QC, Canada, in 1995 and the M.A.Sc. and Ph.D. degrees in electrical and computer engineering from the University of Toronto, Toronto, ON, Canada, in 1997 and 2000, respectively.

Since 2000, he has been with the Photonic Systems Group, Department of Electrical and Computer Engineering, McGill University. During 2006 and 2007, he was on leave with Queen's University, Ryerson University, the Chinese University of Hong Kong, and the Universidad Politécnica de Valencia. His research interests are in ultrafast photonics and fiber optics and include arbitrary optical waveform generation, all-optical signal processing, fiber lasers and amplifiers, and fiber gratings.

Dr. Chen's conference organization activities include serving on the Technical Program Committee for the IEEE-LEOS Annual Meeting and the OSA Topical Meeting on Bragg gratings, photosensitivity, and poling. He is an Associate Editor (Canada) for the IEEE-LEOS Newsletter and a Topical Editor for Optics Letters. 